\title{
Generalized Reliability Analysis of Mechanical Systems with Imperfect Maintenance
}

\author{
Peng Gao $\mathbb{D}^{1}$ and Liyang Xie ${ }^{2}$ \\ ${ }^{1}$ School of Mechanical Engineering, Liaoning Petrochemical University, Fushun, Liaoning 113001, China \\ ${ }^{2}$ Ministry of Education Key Laboratory of Aero Power Equipment Vibration and Control, Northeastern University, Shenyang, \\ Liaoning 110819, China \\ Correspondence should be addressed to Peng Gao; gaopeng@lnpu.edu.cn
}

Received 5 July 2021; Accepted 3 November 2021; Published 10 January 2022

Academic Editor: Elena Zaitseva

Copyright (c) 2022 Peng Gao and Liyang Xie. This is an open access article distributed under the Creative Commons Attribution License, which permits unrestricted use, distribution, and reproduction in any medium, provided the original work is properly cited.

\begin{abstract}
Generalized reliability models and failure rate models of mechanical systems are developed in this paper according to the system working mechanism, which take the design parameters as input. The models consider strength degradation and imperfect maintenance. Besides, the models take into account the failure correlation caused by homologous load effect and the maintenance correlation owing to group maintenance. Unlike traditional reliability models, the models do not rely on empirical assumptions when considering failure correlation and maintenance correlation and have clear physical meaning. Moreover, the correctness and effectiveness of the models are verified by Monte Carlo simulations. Finally, the influences of failure correlation and maintenance correlation on generalized reliability, the influences of failure correlation on maintenance correlation, and the influences of maintenance correlation on failure correlation are analyzed via numerical examples. The results show that failure correlation and maintenance correlation have great influences on generalized reliability, and the interaction between the two correlation shows obvious time-varying characteristics.
\end{abstract}

\section{Introduction}

In order to prevent the serious impact of the failure of mechanical parts under dynamic loads, preventive maintenance, corrective maintenance, other maintenance, and replacement strategies are always carried out in the whole system operation process. The random dynamic degradation behavior of components and systems brings great difficulties to the evaluation of operational status of the components and systems, which also brings great challenges to the formulation of maintenance and replacement strategies. Therefore, it is necessary to establish generalized reliability models of mechanical systems considering both dynamic stochastic degradation $[1,2]$ and maintenance activities.

In the past few decades, researchers have carried out a lot of in-depth and effective research on system reliability assessment based on these models. For instance, Wang proposed a fuzzy stress-strength interference model with the line-segment method [3]. An represented a discrete reliability model of mechanical components to deal with the problem that the stress cannot be regarded as a single random variable when the components worked in multioperating conditions [4]. Zhang established a time-varying stress-strength interference model to calculate structural reliability with the time-varying stable model for Kendall's tau used to address the correlation problem in reliability calculation [5]. Barati constructed an improved load-capacity interference model which can be used to assess reliability in the case of lack of sufficient failure data in specific operating conditions [6]. A system-level load-strength interference model was introduced by $\mathrm{Wu}$ considering dependent failure and multiple failure models for reliability estimation of shiplift gear systems [7]. Yan applied the stress-strength interference model to the reliability analysis and the optimization design of passenger car clutch [8]. In the case where accurate probability density functions of 
stress and strength cannot be available, Guo proposed an interval discrete stress-strength interference model to determine the lower and upper bounds of component reliability [9]. These models lay a foundation for reliability evaluation of mechanical systems under various working conditions and sample conditions.

When serious performance degradation or failure happens to mechanical products, maintenance or replacement has to be performed to ensure the economy and safety of the products [10-15]. Maintenance can be classified into corrective maintenance $(\mathrm{CM})$ and preventive maintenance (PM). It is very difficult to predict the time-varying statistical characteristics of the random degradation process according to the system design parameters and environmental loads, which brings great challenges in developing generalized reliability models and providing guidance for PM. Meanwhile, statistical correlation of degradation and failure between different components in a system, which is referred to as failure dependence, is always encountered, which makes the independent assumption about component failure invalid, resulting in the invalidity of the bottom-up system reliability modeling method.

In the process of $\mathrm{CM}$ or $\mathrm{PM}$, the actual completion of maintenance behavior is uncertain [16-19], which is described by the concept of imperfect maintenance. Moreover, owing to the maintenance habits of maintenance personnel and the share of setting up and tools, maintenance dependence could occur, which makes the states of components after a repair statistically correlative. The joint influences of failure dependence, imperfect maintenance, and maintenance dependence make the system state prediction and generalized reliability prediction significantly difficult. Currently, the mechanism of the joint influences lacks further explanation, and the quantitative generalized reliability models considering the joint influences are seldom reported.

To address the abovementioned problems, time-varying reliability models of mechanical systems components are developed with the damage caused by random loads considered. The models can consider the failure dependence and be used to derive time-varying failure rate models. Furthermore, according to the stochastic degradation characteristics of the mechanical systems, the generalized timevarying reliability models considering imperfect maintenance are established.

\section{Statistical Correlation in Generalized Reliability Estimation}

As mentioned above, reliability can be divided into narrow sense reliability and generalized reliability according to whether maintenance behavior is considered or not. For the convenience of description, narrow sense reliability is referred to as NSR, and generalized reliability is referred to as GR. Failure correlation and maintenance correlation are key and difficult issues in NSR analysis and GR analysis, respectively. In the current literature, failure correlation brings a large challenge in system NSR analysis as shown in Figure 1, especially for mechanical systems. To deal with the

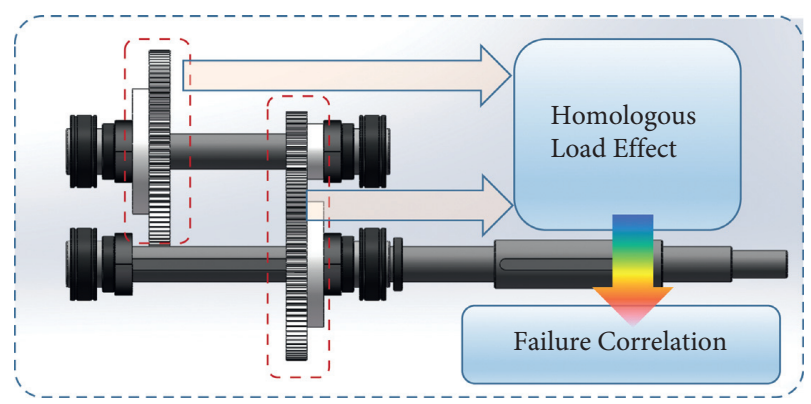

FIGURE 1: Failure correlation from homologous load effects in NSR assessment.

problem of failure correlation, failure rates of the components are always used under the assumption that they are statistically independent. Then, the system failure rate is obtained from the system structure function by correcting these failure rates of the components with empirical coefficients. Typical models include the $\beta$ factor model [20], the alpha factor model [21], the multiple Greek model [22], and the binomial failure rate model.

Maintenance correlation results in large challenges in GR analysis. Maintenance correlation mainly includes (1) Structure correlation. It refers to the correlation caused by system working mechanism and maintenance process [23]. For instance, disassembly of a component causes the disassembly of other related components. (2) Different from the independent maintenance of a single part, components in group maintenance are more likely to be statistically correlative. In this case, maintenance correlation may take the following forms: the economic correlation, the maintenance effect correlation caused by operation habits and maintenance experience of maintenance personnel in group maintenance, and the maintenance effect correlation caused by using the same maintenance equipment as shown in Figure 2 .

Generally, failure correlation and maintenance correlation are discussed independently in NSR analysis and GR analysis, respectively, and there are few quantitative studies on the relationship between them according to the working mechanism of mechanical systems.

As a matter of fact, for mechanical systems, failure correlation is closely related to maintenance correlation. For example, as shown in Figure 3, due to the randomness of initial strength, the strength degradation paths could be random. To represent the randomness of the paths, two schematic possible paths (Path1 and Path 2) in work cycle 1 are listed in Figure 3. The failure or degradation of components in a mechanical system has a statistical correlation due to the influence of the same source load in the working process of the mechanical system. In the process of PM, when there exists maintenance correlation, the recovery level of the components will be highly correlated. To illustrate the randomness of the maintenance effect, each residual strength in Path 1 and Path 2 at the end of work cycle 1 could restore to two different possible states that evolve into two possible paths in work cycle 2 . The situation of the possible strength degradation paths in work cycle 3 is 


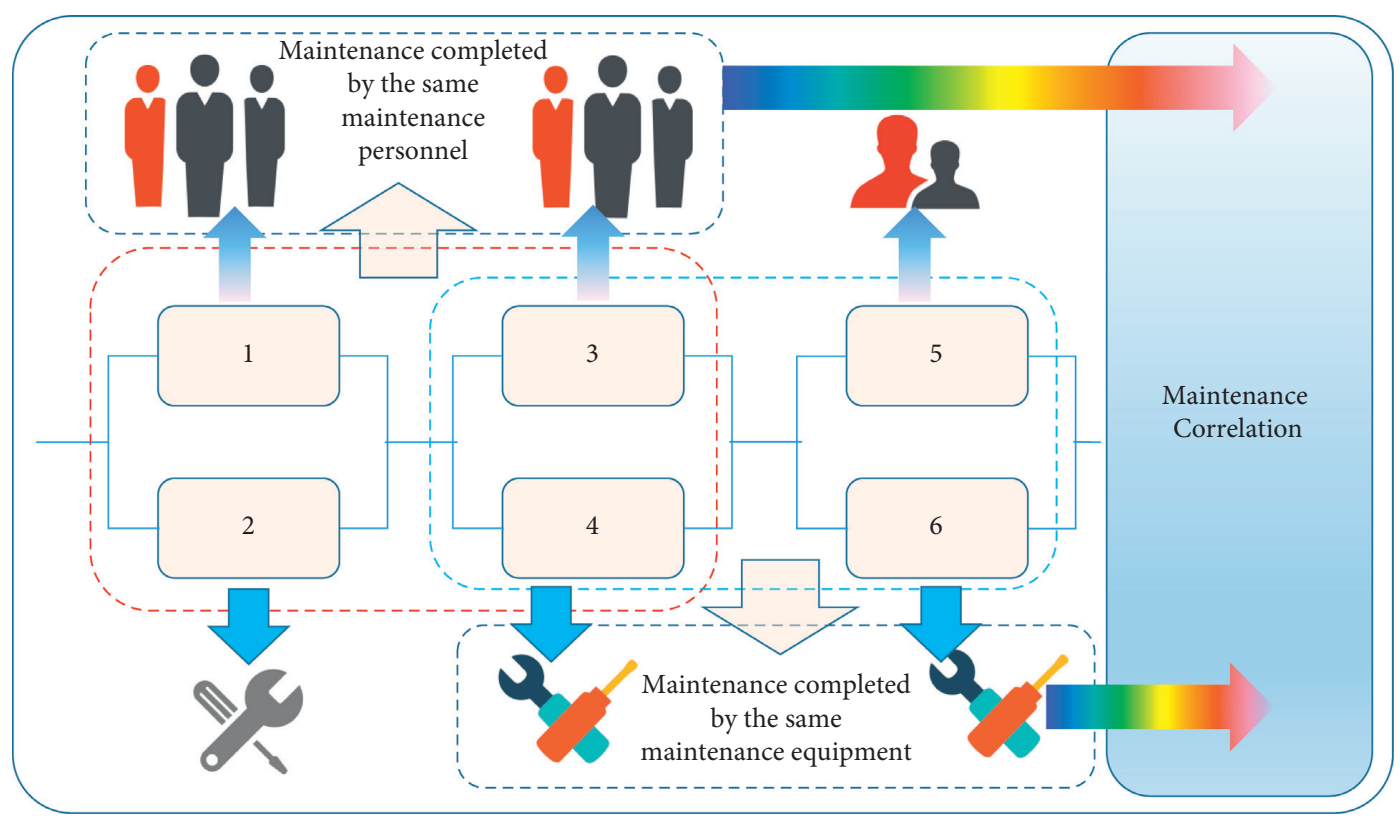

FIGURE 2: Maintenance correlation in GR assessment.

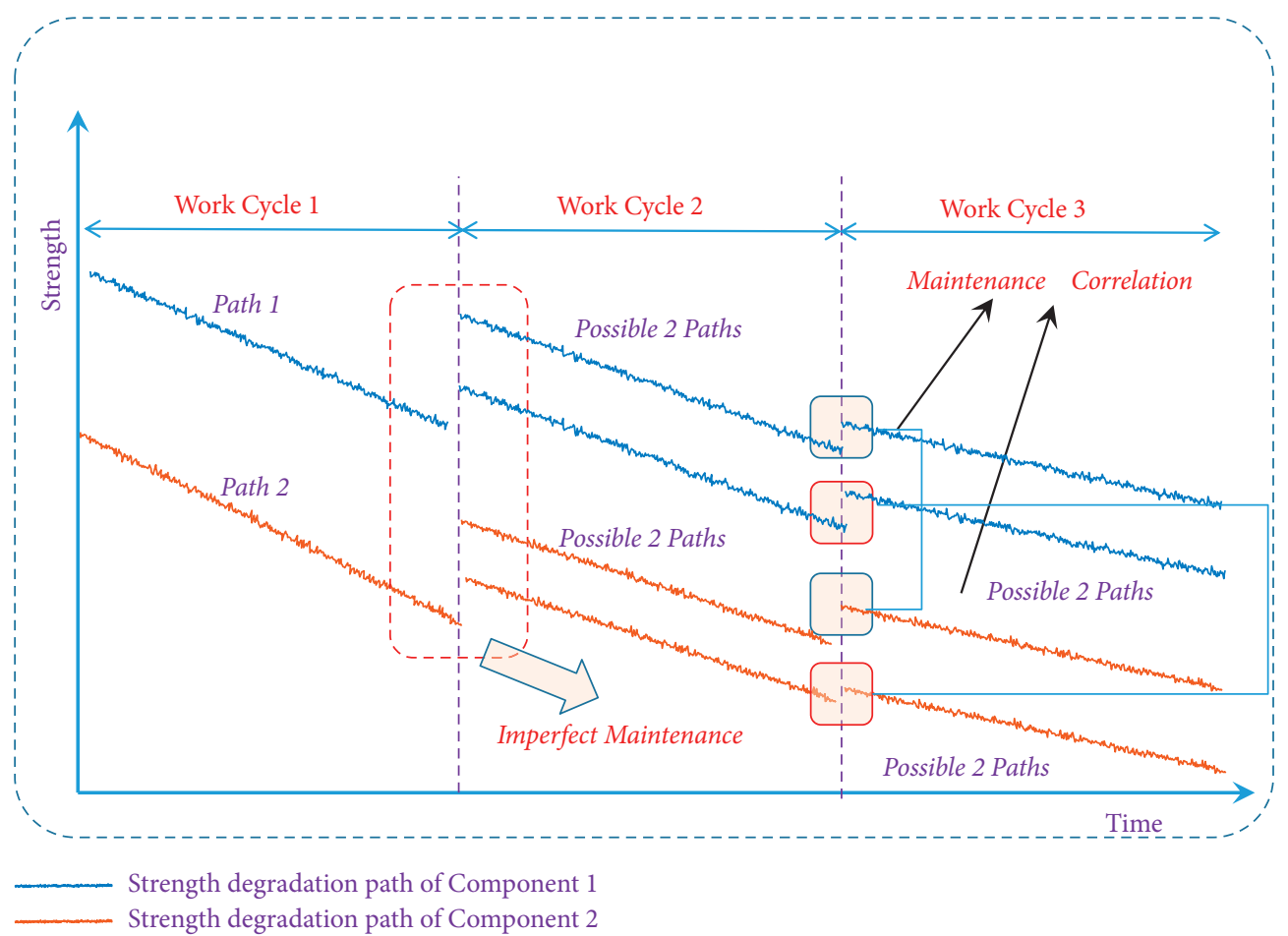

FIGURE 3: Strength degradation of components considering maintenance correlation.

similar to that of work cycle 2. Because of the effect of homologous load, these components will continue to have a high degree of failure correlation in work cycle 2 and work cycle 3. Meanwhile, the increase of failure correlation increases the possibility of simultaneous failure and synchronous degradation, which could result in maintenance correlation. Hence, it is quite imperative and challenging to model the interaction of the two effects and establish GR models of mechanical systems with the two effects taken into consideration.
In addition, constant failure rate seldom occurs for mechanical components and mechanical systems, and most mechanical products are expensive. Hence, it is almost impossible to obtain the failure rate of components and systems through destructive tests. Therefore, it is not feasible to use the failure rate of components as a parameter to construct GR models of mechanical systems. It is necessary to describe the failure correlation and maintenance correlation in essence based on the working mechanism and 
mechanical design parameters and further establish GR models considering the two effects.

\section{System Reliability Analysis}

3.1. NSR Modeling. For a component working under the failure mode of fatigue, the alternating action of random load causes the random degradation of strength. Because the components in the mechanical systems often undertake the task of transmitting power, most of the mechanical systems can be classified into series systems logically. In this paper, we will consider the NSR modeling and GR modeling of series mechanical systems. Considering a mechanical system composed of $N$ components, the stress $s_{\mathrm{ij}}\left(L_{j}\right)$ of the $i$ th $(i=1$, $2, \ldots, n)$ component subjected to the $j$ th application of deterministic working load $L$ can be obtained from the system stress matrix $\mathbf{S}$ in the discrete element by the finite element method as follows [24]:

$$
\begin{aligned}
& L=K \cdot q, \\
& S=E \cdot \varepsilon, \\
& \varepsilon=B \cdot q,
\end{aligned}
$$

where $L$ is the system external force matrix, $K$ is the system stiffness matrix, $E$ is the elastic modulus matrix, $\varepsilon$ is the strain matrix, $\mathbf{B}$ is the strain-displacement matrix and $q$ is the nodal displacement matrix.

Consider the strength $r_{\mathrm{ij}}$ of the component at the load application and the distribution of the stress $s_{\mathrm{ij}}\left(L_{j}\right)$ characterized by the probability density function (PDF) of $f\left(s_{\mathrm{ij}}\left(L_{j}\right)\right)$, its NSR can be expressed as

$$
R_{\mathrm{ij}}=\int_{-\infty}^{\infty} f\left(s_{\mathrm{ij}}\left(L_{j}\right)\right)\left(r_{\mathrm{ij}}-s_{\mathrm{ij}}\left(L_{j}\right)\right) \mathrm{d} s_{\mathrm{ij}}\left(L_{j}\right),
$$

where $(*)$ is the indicator function as follows and the reliability calculated can be completed by using the numerical solution technique.

$$
(z)=\left\{\begin{array}{ll}
1, & z \geq 0 \\
0, & z<0
\end{array} .\right.
$$

Provided that the number of load application times $n$ is taken into account, the component NSR can be given by

$$
R_{\mathrm{ij}}(n)=\prod_{j=1}^{n} \int_{-\infty}^{\infty} f\left(s_{\mathrm{ij}}\left(L_{j}\right)\right)\left(r_{\mathrm{ij}}-s_{\mathrm{ij}}\left(L_{j}\right)\right) \mathrm{d} s_{\mathrm{ij}}\left(L_{j}\right) .
$$

For statistically independent mechanical components in a mechanical system, according to the reliability theory of series systems, the system NSR is expressed from equation (4) as follows:

$$
R_{\text {insys } 1}(n)=\prod_{i=1}^{N} \prod_{j=1}^{n} \int_{-\infty}^{\infty} f\left(s_{\mathrm{ij}}\left(L_{j}\right)\right)\left(r_{\mathrm{ij}}-s_{\mathrm{ij}}\left(L_{\mathrm{ij}}\right)\right) \mathrm{d} s_{\mathrm{ij}}\left(L_{j}\right) .
$$

Due to the randomness of material defects and manufacturing process, the initial strength of mechanical components always presents the characteristics of randomness. In this paper, the randomness of the initial strength of the $N$ components is expressed by the PDF of $f_{i}\left(r_{i}\right)(i=1,2, \ldots, N)$. The strength degradation path of the components and the corresponding equivalent residual strength $r_{\mathrm{ij}}$ can be obtained via the authors' previous work in the literature [25].

Then, the system NSR expression can be further obtained as follows:

$$
R_{\text {insys }}(n)=\prod_{i=1}^{N}\left[\int_{-\infty}^{\infty} f_{i}\left(r_{i}\right) \prod_{j=1}^{n} \int_{-\infty}^{\infty} f\left(s_{\mathrm{ij}}\left(L_{j}\right)\right)\left(r_{\mathrm{ij}}-s_{\mathrm{ij}}\left(L_{j}\right)\right) \mathrm{d} s_{\mathrm{ij}}\left(L_{j}\right) \mathrm{d} r_{i}\right] .
$$

The corresponding system failure rate (FR) can be expressed as

$$
\begin{aligned}
\phi_{\text {insys }}(n)= & \left\{\prod_{i=1}^{N}\left[\int_{-\infty}^{\infty} f_{i}\left(r_{i}\right) \prod_{j=1}^{n} \int_{-\infty}^{\infty} f\left(s_{\mathrm{ij}}\left(L_{j}\right)\right)\left(r_{\mathrm{ij}}-s_{\mathrm{ij}}\left(L_{j}\right)\right) \mathrm{d}_{\mathrm{ij}}\left(L_{j}\right) \mathrm{d} r_{i}\right]\right. \\
& -\frac{\prod_{i=1}^{N}\left[\int_{-\infty}^{\infty} f_{i}\left(r_{i}\right) \prod_{j=1}^{n+1} \int_{-\infty}^{\infty} f\left(s_{\mathrm{ij}}\left(L_{j}\right)\right)\left(r_{\mathrm{ij}}-s_{\mathrm{ij}}\left(L_{j}\right)\right) \mathrm{d} s_{\mathrm{ij}}\left(L_{j}\right) \mathrm{d} r_{i}\right]}{\left\{\prod_{i=1}^{N}\left[\int_{-\infty}^{\infty} f_{i}\left(r_{i}\right) \prod_{j=1}^{n} \int_{-\infty}^{\infty} f\left(s_{\mathrm{ij}}\left(L_{j}\right)\right)\left(r_{\mathrm{ij}}-s_{\mathrm{ij}}\left(L_{j}\right)\right) \mathrm{d} \mathrm{s}_{\mathrm{ij}}\left(L_{j}\right) \mathrm{d} r_{i}\right]\right\}} .
\end{aligned}
$$


As a matter of fact, the stress on each component is obtained through the joint external load. Thus, the statistical characteristics of all stress are determined by the statistical characteristics of the joint external load, which is defined as homologous load effect (HLE). In this case, failure correlation may occur, and the system NSR could be significantly affected. When considering the HLE and the distribution of the joint working load characterized by the PDF of $f\left(L_{j}\right)$, the system NSR can be expressed as

$$
R_{\text {sys }}(n)=\int_{-\infty}^{\infty} f_{1}\left(r_{1}\right) \int_{-\infty}^{\infty} f_{2}\left(r_{2}\right) \cdots \int_{-\infty}^{\infty} f_{N}\left(r_{N}\right)\left[\prod_{j=1}^{n} \int_{-\infty}^{\infty} f\left(L_{j}\right)\left[\prod_{i=1}^{N}\left[\left(r_{\mathrm{ij}}-s_{\mathrm{ij}}\left(L_{j}\right)\right)\right]\right] \mathrm{d} L_{j}\right] \mathrm{d} r_{1} \mathrm{~d} r_{2} \cdots \mathrm{d} r_{N} .
$$

The corresponding system FR considering the HLE can be given by

$$
\begin{aligned}
\phi_{\text {sys }}(n)= & \int_{-\infty}^{\infty} f_{1}\left(r_{1}\right) \int_{-\infty}^{\infty} f_{2}\left(r_{2}\right) \cdots \int_{-\infty}^{\infty} f_{N}\left(r_{N}\right)\left\{\left[\prod_{j=1}^{n} \int_{-\infty}^{\infty} f\left(L_{j}\right)\left[\prod_{i=1}^{N}\left[\left(r_{\mathrm{ij}}-s_{\mathrm{ij}}\left(L_{j}\right)\right)\right]\right] \mathrm{d} \mathrm{L}_{j}\right]\right\} \\
& \frac{\left[\prod_{j=1}^{n+1} \int_{-\infty}^{\infty} f\left(L_{j}\right)\left[\prod_{i=1}^{N}\left[\left(r_{\mathrm{ij}}-s_{\mathrm{ij}}\left(L_{j}\right)\right)\right]\right] \mathrm{dL} \mathrm{L}_{j}\right] \mathrm{d} r_{1} \mathrm{~d} r_{2} \cdots \mathrm{d} r_{N}}{\left\{\int_{-\infty}^{\infty} f_{1}\left(r_{1}\right) \int_{-\infty}^{\infty} f_{2}\left(r_{2}\right) \cdots\left[\prod_{j=1}^{n} \int_{-\infty}^{\infty} f\left(L_{j}\right)\left[\prod_{i=1}^{N}\left[\left(r_{\mathrm{ij}}-s_{\mathrm{ij}}\left(L_{j}\right)\right)\right]\right] \mathrm{d} L_{j}\right] \mathrm{d} r_{1} \mathrm{~d} r_{2} \cdots \mathrm{d} r_{N}\right\}} .
\end{aligned}
$$

3.2. GR Modeling with Independent Maintenance. In this section, the maintenance correlation mechanism is analyzed, and GR models are developed considering both the maintenance correlation and failure correlation based on the NSR models proposed in Section 3.1. The proposed GR models can be used to quantitatively analyze the joint influences of maintenance correlation and failure correlation on system GR. Besides, the models can also be used to describe the relationship between the failure correlation and the maintenance correlation.

In order to prevent failure of mechanical systems caused by strength degradation and impact load, it is necessary to carry out PM. To consider the working mechanism of mechanical systems and avoid the shortcomings of unclear physical meaning in the empirical parameter models, the GR models use the residual strength of components at each preventive as the index to reflect the maintenance effect. The significance of using residual strength as the index lies in the following: (1) The index is a key parameter in NSR modeling, which is important to characterize the failure correlation. (2) The change of the index can not only characterize the maintenance effect but also reveal the maintenance correlation in essence. (3) The index is an important design parameter for mechanical products. The degradation mechanism and the working mechanism of mechanical systems can be characterized by this index. Therefore, the index works before and after maintenance, which can reveal the relationship between failure correlation and maintenance correlation. Furthermore, it has clear physical meaning, which helps to avoid empirical hypothesis in the process of modeling.

In this paper, for the convenience of description, it is assumed that PM will be carried out after a mechanical system completes a work cycle. In the $k$ th work cycle, the working load applies for $n_{k}$ times, and the residual strength of the $i$ th component after the $k$ th work cycle is denoted by $r_{\mathrm{in}_{k}}^{k}$. After the $k$ th PM, the residual strength is improved. The relationship between the residual strength before and after maintenance is expressed as:

$$
r_{i}^{k+1}=\propto_{i}^{k} r_{\mathrm{in}_{k}}^{k},
$$

where $r_{i}^{k+1}$ is the initial strength of the $i$ th component in the $(k+1)$ th work cycle. $\propto_{i}^{k}$ is defined as the maintenance ratio (MR), which is the ratio of residual strength before and after maintenance and represents the maintenance effect.

To characterize the relationship between the initial strength of the $i$ th component at the beginning of the $k$ th work cycle and its initial strength at the beginning of the first work cycle, the maintenance factors (MFs) $\beta_{i}^{k}$ are defined as follows:

$$
r_{i}^{k}=\beta_{i}^{k} r_{i} \text {. }
$$

Then, the relationship between $r_{i}$ and $r_{\mathrm{in}_{k}}^{k}$ can be given by

$$
r_{\mathrm{in}_{k}}^{k}=\frac{\beta_{i}^{k+1}}{\propto_{i}^{k}} r_{i}=\gamma^{k} r_{i},
$$

where $\gamma^{k}=\beta_{i}^{k+1} / \propto_{i}^{k}$.

$\gamma^{k}$ is defined as the performance degradation parameter (PDP). The MF can be used to illustrate the recovery of strength at the beginning of each work cycle, which mainly depends on the maintenance capability. The PDP reflects the joint cumulative effect of maintenance activities and the damage resulting from the working load. Generally, it should be satisfied that 


$$
\gamma^{k-1} \leq \beta_{i}^{k} \leq 1
$$

In particular, if $\beta_{i}^{k}=1$, the component recovers to the original state, and the maintenance is essentially a perfect maintenance. If $\beta_{i}^{k}=\gamma^{k-1}$, the component maintains the performance level before maintenance, and the maintenance is essentially a minimum maintenance. When the MF lies between these two extremes, the performance of the component has been improved but not to the original level, which can be regarded as an imperfect maintenance. In practice, owing to the randomness of both the maintenance behavior of maintenance personnel and the tools, the maintenance effect could be random.

To deal with the randomness, the distribution of the MF characterized by the PDF of the MF of $f\left(\beta_{i}^{k}\right)$ is adopted in this paper. Then, the GR of the $i$ th component within the $(k+1)$ th work cycle after the $k$ th maintenance with the load application for $n^{k+1}$ times can be expressed by

$$
R_{\mathrm{ij}}^{k+1}\left(n^{k+1}\right)=\int_{-\infty}^{\infty} f\left(\beta_{i}^{k+1}\right) \prod_{j=1}^{n^{k+1}} \int_{-\infty}^{\infty} f\left(s_{\mathrm{ij}}^{k+1}\left(L_{j}^{k+1}\right)\right)\left(r_{\mathrm{ij}}^{k+1}\left(\beta_{i}^{k+1}\right)-s_{\mathrm{ij}}^{k+1}\left(L_{j}^{k+1}\right)\right) \mathrm{d} s_{\mathrm{ij}}^{k+1}\left(L_{j}^{k+1}\right) d \beta_{i}^{k+1},
$$

where $L_{j}^{k+1}$ is the $j$ th work load in the $(k+1)$ th work cycle, $s_{\mathrm{ij}}^{k+1}$ is the stress on the $i$ th component under the application of $L_{j}^{k+1}$, and $r_{\mathrm{ij}}^{k+1}$ is the residual strength at the $j$ th application of $L_{j}^{k+1}$ with the initial strength in the $(k+1)$ th work cycle of $\beta_{i}^{k+1} r_{i}$. For a mechanical system with statistically independent components, the GR in the $(k+1)$ th work cycle can derived from equation (14) as follows:

$$
\begin{aligned}
R_{\text {insys }}^{k+1}\left(n^{k+1}\right)= & \prod_{i=1}^{N} \int_{-\infty}^{\infty} f\left(\beta_{i}^{k+1}\right) \prod_{j=1}^{n^{k+1}} \int_{-\infty}^{\infty} f\left(s_{\mathrm{ij}}^{k+1}\left(L_{j}^{k+1}\right)\right) o\left(r_{\mathrm{ij}}^{k+1}\left(\beta_{i}^{k+1}\right)-s_{\mathrm{ij}}^{k+1}\left(L_{j}^{k+1}\right)\right) \\
& \mathrm{d} s_{\mathrm{ij}}^{k+1}\left(L_{j}^{k+1}\right) d \beta_{i}^{k+1} .
\end{aligned}
$$

The corresponding system generalized failure rate (GFR) in the $(k+1)$ th work cycle can be given by:

$$
\begin{aligned}
\varphi_{\mathrm{insys}}^{k+1}\left(n^{k+1}\right)= & \prod_{i=1}^{N} \int_{-\infty}^{\infty} f\left(\beta_{i}^{k+1}\right) \prod_{j=1}^{n^{k+1}} \int_{-\infty}^{\infty} f\left(s_{\mathrm{ij}}^{k+1}\left(L_{j}^{k+1}\right)\right)\left(r_{\mathrm{ij}}^{k+1}\left(\beta_{i}^{k+1}\right)-s_{\mathrm{ij}}^{k+1}\left(L_{j}^{k+1}\right)\right) \mathrm{d} s_{\mathrm{ij}}^{k+1}\left(L_{j}^{k+1}\right) d \beta_{i}^{k+1}- \\
& \frac{\prod_{i=1}^{N} \int_{-\infty}^{\infty} f\left(\beta_{i}^{k+1}\right) \prod_{j=1}^{n^{k+1}+1} \int_{-\infty}^{\infty} f\left(s_{i j}^{k+1}\left(L_{j}^{k+1}\right)\right)\left(r_{\mathrm{ij}}^{k+1}\left(\beta_{i}^{k+1}\right)-s_{\mathrm{ij}}^{k+1}\left(L_{j}^{k+1}\right)\right) \mathrm{d} s_{\mathrm{ij}}^{k+1}\left(L_{j}^{k+1}\right) d \beta_{i}^{k+1}}{} \\
& \left\{\prod_{i=1}^{N} \int_{-\infty}^{\infty} f\left(\beta_{i}^{k+1}\right) \prod_{j=1}^{n^{k+1}} \int_{-\infty}^{\infty} f\left(s_{\mathrm{ij}}^{k+1}\left(L_{j}^{k+1}\right)\right)\left(r_{\mathrm{ij}}^{k+1}\left(\beta_{i}^{k+1}\right)-s_{\mathrm{ij}}^{k+1}\left(L_{j}^{k+1}\right)\right) \mathrm{d} s_{\mathrm{ij}}^{k+1}\left(L_{j}^{k+1}\right) d \beta_{i}^{k+1}\right\}
\end{aligned}
$$

The GR of the system in all $n_{\text {all }}$ work cycles considering the maintenance activities can be written as:

$$
\begin{aligned}
R_{\mathrm{insys}}^{n_{\mathrm{all}}}= & \prod_{i=1}^{N} \int_{-\infty}^{\infty} f_{i}\left(r_{i}\right)\left\{\left[\prod_{j=1}^{n_{1}} \int_{-\infty}^{\infty} f\left(s_{\mathrm{ij}}^{1}\left(L_{j}^{1}\right)\right)\left(r_{\mathrm{ij}}^{1}-s_{\mathrm{ij}}^{1}\left(L_{j}^{1}\right)\right) \mathrm{d} s_{\mathrm{ij}}^{1}\left(L_{j}^{1}\right)\right]\right\} \\
& \prod_{k=1}^{n_{\mathrm{alll}}-1}\left[\int_{-\infty}^{\infty} f\left(\beta_{i}^{k+1}\right) \prod_{j=1}^{n_{k+1}} \int_{-\infty}^{\infty} f\left(s_{\mathrm{ij}}^{k+1}\left(L_{j}^{k+1}\right)\right)\left(r_{\mathrm{ij}}^{k+1}\left(\beta_{i}^{k+1}\right)-s_{\mathrm{ij}}^{k+1}\left(L_{j}^{k+1}\right)\right) \mathrm{d} s_{\mathrm{ij}}^{k+1}\left(L_{j}^{k+1}\right) d \beta_{i}^{k+1}\right] \mathrm{d} r_{i} .
\end{aligned}
$$

When considering the HLE and the distribution of the joint working load characterized by the PDF of $f\left(L_{j}^{k+1}\right)$, the system GR in the $(k+1)$ th work cycle can be obtained as follows: 


$$
\begin{aligned}
R_{\mathrm{sys}}^{k+1}\left(n^{k+1}\right)= & \int_{-\infty}^{\infty} f\left(\beta_{1}^{k+1}\right) \int_{-\infty}^{\infty} f\left(\beta_{2}^{k+1}\right) \cdots \int_{-\infty}^{\infty} f\left(\beta_{N}^{k+1}\right) \\
& \left\{\prod_{j=1}^{n^{k+1}}\left[\int_{-\infty}^{\infty} f\left(L_{j}^{k+1}\right)\left[\prod_{i=1}^{N}\left[o\left(r_{\mathrm{ij}}^{k+1}\left(\beta_{i}^{k+1}\right)-s_{\mathrm{ij}}^{k+1}\left(L_{j}^{k+1}\right)\right)\right]\right] \mathrm{d} L_{j}^{k+1}\right]\right\} d \beta_{1}^{k+1} d \beta_{2}^{k+1} \cdots d \beta_{N}^{k+1} .
\end{aligned}
$$

The corresponding system GFR in the $(k+1)$ th work cycle is

$$
\begin{aligned}
& \varphi_{\text {sys }}^{k+1}\left(n^{k+1}\right)=\int_{-\infty}^{\infty} f\left(\beta_{1}^{k+1}\right) \int_{-\infty}^{\infty} f\left(\beta_{2}^{k+1}\right) \cdots \int_{-\infty}^{\infty} f\left(\beta_{N}^{k+1}\right) \\
& \left\{\prod_{j=1}^{n^{k+1}}\left[\int_{-\infty}^{\infty} f\left(L_{j}^{k+1}\right)\left[\prod_{i=1}^{N}\left[\left(r_{\mathrm{ij}}^{k+1}\left(\beta_{i}^{k+1}\right)-s_{\mathrm{ij}}^{k+1}\left(L_{j}^{k+1}\right)\right)\right]\right] \mathrm{d} L_{j}^{k+1}\right]-\prod_{j=1}^{n^{k+1}+1}\left[\int_{-\infty}^{\infty} f\left(L_{j}^{k+1}\right)\left[\prod_{i=1}^{N}\left[\left(r_{\mathrm{ij}}^{k+1}\left(\beta_{i}^{k+1}\right)-s_{\mathrm{ij}}^{k+1}\left(L_{j}^{k+1}\right)\right)\right]\right] \mathrm{d} L_{j}^{k+1}\right]\right\} \\
& \frac{d \beta_{1}^{k+1} d \beta_{2}^{k+1} \cdots d \beta_{N}^{k+1}}{\left\{\int_{-\infty}^{\infty} f\left(\beta_{1}^{k+1}\right) \int_{-\infty}^{\infty} f\left(\beta_{2}^{k+1}\right) \cdots \int_{-\infty}^{\infty} f\left(\beta_{N}^{k+1}\right)\left\{\prod_{j=1}^{n^{k+1}}\left[\int_{-\infty}^{\infty} f\left(L_{j}^{k+1}\right)\left[\prod_{i=1}^{N}\left[\left(r_{\mathrm{ij}}^{k+1}\left(\beta_{i}^{k+1}\right)-s_{\mathrm{ij}}^{k+1}\left(L_{j}^{k+1}\right)\right)\right]\right] \mathrm{d} L_{j}^{k+1}\right]\right\} d \beta_{1}^{k+1} d \beta_{2}^{k+1} \cdots d \beta_{N}^{k+1}\right\}}
\end{aligned}
$$

The system GR in all $n_{\text {all }}$ work cycles considering the HLE and the maintenance activities can be written as

$$
\begin{aligned}
R_{\text {insys }}^{n_{\text {all }}}= & \int_{-\infty}^{\infty} f_{1}\left(r_{1}\right) \int_{-\infty}^{\infty} f_{2}\left(r_{2}\right) \cdots \int_{-\infty}^{\infty}\left\{\prod_{j=1}^{n_{1}} \int_{-\infty}^{\infty} f\left(L_{j}^{1}\right)\left[\prod_{i=1}^{N}\left[\left(r_{\mathrm{ij}}^{1}-s_{\mathrm{ij}}^{1}\left(L_{j}^{1}\right)\right)\right]\right] \mathrm{d} L_{j}^{1}\right\} \\
& \left\{\prod _ { k = 1 } ^ { n _ { \text { all } } - 1 } \int _ { - \infty } ^ { \infty } f ( \beta _ { 1 } ^ { k + 1 } ) \int _ { - \infty } ^ { \infty } f ( \beta _ { 2 } ^ { k + 1 } ) \cdots \int _ { - \infty } ^ { \infty } f ( \beta _ { N } ^ { k + 1 } ) \left\{\prod_{j=1}^{n_{k+1}} \int_{-\infty}^{\infty} f\left(L_{j}^{k+1}\right)\left[\prod_{i=1}^{N}\left[\left(r_{\mathrm{ij}}^{k+1}\left(\beta_{i}^{k+1}\right)-s_{\mathrm{ij}}^{k+1}\left(L_{j}^{k+1}\right)\right)\right] \mathrm{d} L_{j}^{k+1}\right\}\right.\right. \\
& \left.d \beta_{1}^{k+1} d \beta_{2}^{k+1} \cdots d \beta_{N}^{k+1}\right\} \mathrm{d} r_{1} \mathrm{~d} r_{2} \cdots \mathrm{d} r_{N} .
\end{aligned}
$$

3.3. GR Modeling considering Maintenance Correlation. The models in Section 3.2 are proposed with the maintenance activities for each component mutually statistically independent. However, due to the adoption of the group maintenance strategy and the synchronous damage of multiple components caused by failure correlation, it always occurs that a repairman uses the same equipment to repair a group of components. In this case, the maintenance experience, maintenance habits of maintenance personnel, and the accuracy ability of the equipment itself may cause the same or similar maintenance effect of the components to be repaired, which is a typical maintenance correlation. In order to quantitatively describe the correlation, it is assumed that the components to be repaired are divided into $\mathrm{H}$ groups, and the maintenance effect of the Ith group is expressed by the common MF PDF of $f\left(\beta_{\text {group I }}^{k+1}\right)$. Then, for the mechanical system with independent components, the reliability of the system considering the maintenance correlation in the $(k+1)$ th work cycle can be expressed as

$$
R_{\text {MCinsys }}^{k+1}\left(n^{k+1}\right)=\prod_{I=1}^{H} \int_{-\infty}^{\infty} f\left(\beta_{\text {groupI }}^{k+1}\right) \prod_{i=1}^{g_{I}} \prod_{j=1}^{n^{k+1}} \int_{-\infty}^{\infty} f\left(s_{\text {grouplij }}^{k+1}\left(L_{j}^{k+1}\right)\right)\left(r_{\text {grouplij }}^{k+1}\left(\beta_{\text {groupI }}^{k+1}\right)-s_{\text {grouplij }}^{k+1}\left(L_{j}^{k+1}\right)\right) \mathrm{d} s_{\text {grouplij }}^{k+1}\left(L_{j}^{k+1}\right) d \beta_{\text {groupI }}^{k+1}
$$


8

Mathematical Problems in Engineering

where $*{ }_{\text {groupIij }}^{k+1}$ represents the parameter for the $i$ th $(i=1$, $\left.2, \ldots, g_{I}\right)$ component in group $I$ under the $j$ th load application.
The corresponding GFR considering the maintenance correlation in the $(k+1)$ th work cycle can be given by

$$
\begin{aligned}
& \varphi_{\text {MCinsys }}^{k+1}\left(n^{k+1}\right)
\end{aligned}
$$

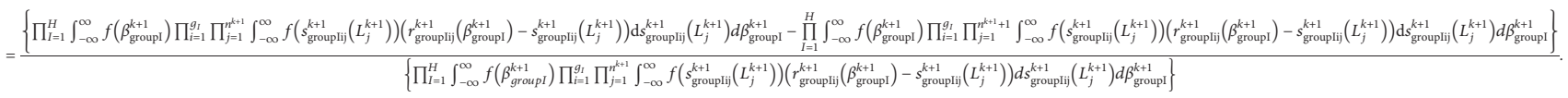

The GR of the system in all $n_{\text {all }}$ work cycles considering the maintenance correlation can be written as:

$$
\begin{aligned}
R_{\mathrm{MCinsys}}^{n_{\text {all }}=} & \prod_{I=1}^{H} \prod_{i=1}^{g_{I}} \int_{-\infty}^{\infty} f_{\text {group Ii }}\left(r_{\text {group Ii }}\right)\left\{\left[\prod_{j=1}^{n_{1}} \int_{-\infty}^{\infty} f\left(s_{\text {groupIij }}^{1}\left(L_{j}^{1}\right)\right)\left(r_{\text {groupIij }}^{1}-s_{\text {groupIij }}^{1}\left(L_{j}^{1}\right)\right) \mathrm{d} s_{\text {grouplij }}^{1}\left(L_{j}^{1}\right)\right]\right\} \\
& \prod_{k=1}^{n_{\text {all }}-1}\left[\int_{-\infty}^{\infty} f\left(\beta_{\text {group }}^{k+1}\right) \prod_{j=1}^{n_{k+1}} \int_{-\infty}^{\infty} f\left(s_{\text {grouplij }}^{k+1}\left(L_{j}^{k+1}\right)\right)\left(r_{\text {grouplij }}^{k+1}\left(\beta_{\text {group }}^{k+1}\right)-s_{\text {grouplij }}^{k+1}\left(L_{j}^{k+1}\right)\right) \mathrm{d} s_{\text {grouplij }}^{k+1}\left(L_{j}^{k+1}\right) d \beta_{\text {group }}^{k+1}\right] \mathrm{d} r_{\text {group Ii }}
\end{aligned}
$$

When considering the HLE and the distribution of the joint working load characterized by the PDF of $f\left(L_{j}^{k+1}\right)$, the system GR considering the maintenance correlation in the $(k+1)$ th work cycle can be obtained as follows:

$$
\begin{aligned}
R_{\text {MCsys }}^{k+1}\left(n^{k+1}\right)= & \int_{-\infty}^{\infty} f\left(\beta_{\text {group } 1}^{k+1}\right) \int_{-\infty}^{\infty} f\left(\beta_{\text {group 2 }}^{k+1} \cdots \int_{-\infty}^{\infty} f\left(\beta_{\text {group H }}^{k+1}\right)\right. \\
& \left\{\prod_{j=1}^{n^{k+1}}\left[\int_{-\infty}^{\infty} f\left(L_{j}^{k+1}\right)\left[\prod_{I=1}^{H} \prod_{i=1}^{g_{I}}\left[\left(r_{\text {group Iii }}^{k+1}\left(\beta_{\text {group }}^{k+1}\right)-s_{\text {group Ii }}^{k+1}\left(L_{j}^{k+1}\right)\right)\right]\right] \mathrm{d} L_{j}^{k+1}\right]\right\} d \beta_{\text {group } 1}^{k+1} d \beta_{\text {group } 2}^{k+1} \cdots d \beta_{\text {group H }}^{k+1}
\end{aligned}
$$

The corresponding system GFR in the $(k+1)$ th work cycle is

$$
\begin{aligned}
& \varphi_{\text {MCSys }}^{k+1}\left(n^{k+1}\right)=\int_{-\infty}^{\infty} f\left(\beta_{\text {group 1 }}^{k+1}\right) \int_{-\infty}^{\infty} f\left(\beta_{\text {group } 2}^{k+1}\right) \cdots \int_{-\infty}^{\infty} f\left(\beta_{\text {groups }}^{k+1}\right) \\
& \left\{\prod_{j=1}^{n^{k+1}}\left[\int_{-\infty}^{\infty} f\left(L_{j}^{k+1}\right)\left[\prod_{I=1}^{H} \prod_{i=1}^{g_{I}}\left[\left(r_{\text {group Icj }}^{k+1}\left(\beta_{\text {groups }}^{k+1}\right)-s_{\text {grouplij }}^{k+1}\left(L_{j}^{k+1}\right)\right)\right]\right] \mathrm{d} L_{j}^{k+1}\right]\right. \\
& \left.-\prod_{j=1}^{n^{k+1}+1}\left[\int-\infty \int_{-\infty}^{\infty} f\left(L_{j}^{k+1}\right)\left[\prod_{I=1}^{H} \prod_{i=1}^{g_{I}}\left[\left(r_{\text {grouplij }}^{k+1}\left(\beta_{\text {groups }}^{k+1}\right)-s_{\text {grouplij }}^{k+1}\left(L_{j}^{k+1}\right)\right)\right]\right] \mathrm{d} L_{j}^{k+1}\right]\right\} \\
& \frac{d \beta_{\text {group } 1}^{k+1} d \beta_{\text {group } 2}^{k+1} \cdots d \beta_{\text {group }}^{k+1}}{\left\{\int_{-\infty}^{\infty} f\left(\beta_{\text {group } 1}^{k+1}\right) \int_{-\infty}^{\infty} f\left(\beta_{\text {group } 2}^{k+1}\right) \cdots \int_{-\infty}^{\infty} f\left(\beta_{\text {groups }}^{k+1}\right)\left\{\prod_{j=1}^{h^{k+1}}\left[\int_{-\infty}^{\infty} f\left(L_{j}^{k+1}\right)\left[\prod_{I=1}^{H} \prod_{i=1}^{g_{I}}\left[\left(r_{\text {grouppij }}^{k+1}\left(\beta_{\text {group } 1}^{k+1}\right)-s_{\text {grouplij }}^{k+1}\left(L_{j}^{k+1}\right)\right)\right]\right] d L_{j}^{k+1}\right]\right\} d \beta_{\text {group } 1}^{k+1} d \beta_{\text {group } 2}^{k+1} \cdots d \beta_{\text {group H }}^{k+1}\right\}} .
\end{aligned}
$$


The system GR inall $n_{\text {all }}$ work cycles considering the HLE and the maintenance correlation can be written as:

$$
\begin{aligned}
R_{\text {insys }}^{n_{\text {all }}}= & \int_{-\infty}^{\infty} f_{1}\left(r_{1}\right) \int_{-\infty}^{\infty} f_{2}\left(r_{2}\right) \cdots \int_{-\infty}^{\infty} f_{N}\left(r_{N}\right)\left\{\prod_{j=1}^{n_{1}} \int_{-\infty}^{\infty} f\left(L_{j}^{1}\right)\left[\prod_{i=1}^{N}\left[\left(r_{\mathrm{ij}}^{1}-s_{\mathrm{ij}}^{1}\left(L_{j}^{1}\right)\right)\right]\right] \mathrm{d} L_{j}^{1}\right\} \\
& \left\{\prod_{k=1}^{n_{\text {all }}^{-1}} \int_{-\infty}^{\infty} f\left(\beta_{\text {group } 1}^{k+1}\right) \int_{-\infty}^{\infty} f\left(\beta_{\text {group 2 }}^{k+1}\right) \cdots \int_{-\infty}^{\infty} f\left(\beta_{\text {group H }}^{k+1}\right)\right. \\
& \left.\left\{\prod_{j=1}^{n^{k+1}}\left[\int_{-\infty}^{\infty} f\left(L_{j}^{k+1}\right)\left[\prod_{I=1}^{H} \prod_{i=1}^{g_{I}}\left[\left(r_{\text {grouplij }}^{k+1}\left(\beta_{\text {groupI }}^{k+1}\right)-s_{\text {grouplij }}^{k+1}\left(L_{j}^{k+1}\right)\right)\right]\right] \mathrm{d} L_{j}^{k+1}\right]\right\} d \beta_{\text {group 1 }}^{k+1} d \beta_{\text {group } 2}^{k+1} \cdots d \beta_{\text {groupH }}^{k+1}\right\} \mathrm{d} r_{1} \mathrm{~d} r_{2} \cdots \mathrm{d} r_{N} .
\end{aligned}
$$

\section{Numerical Examples}

In this section, numerical examples are provided to illustrate the proposed models. Besides, Monte Carlo simulations (MCS) are performed to verify the models, and the influences of the failure correlation and maintenance correlation on GR will be analyzed. Consider a system consisting of six identical components. The system operates for four work cycles with three times of maintenance performed at the end of the first three work cycles. In order to characterize the maintenance effect, the discrete distributions of the MFs at each maintenance are listed in Tables 1-3. The stress on each component at each load application follows the normal distribution with the mean value of $450 \mathrm{MPa}$ and the standard deviation of $30 \mathrm{MPa}$. The initial strength of each component also follows the normal distribution with the mean value of $300 \mathrm{MPa}$ and the standard deviation of $20 \mathrm{MPa}$. The material parameters $a, m$, and $C$ are 1,2 , and $6 \times 10^{9} \mathrm{MPa}^{2}$, respectively, with the physical meaning explained in the authors' previous work in the literature [19]. The GR considering the HLE and maintenance correlation in different work cycles is shown in Figure 4. The corresponding GFR is shown in Figure 5. In order to validate the proposed models, the Monte Carlo simulation is carried out with the flow chart shown in Figure 6, and the results are also shown in Figure 4.

The MCS takes into account the randomness of maintenance activities, stress, and strength degradation and is performed based on actual system working process, which is independent of the solution process of the proposed analytical models. Hence, the effectiveness of simulation verification can be ensured. It can be seen from Figure 4 that the simulation results in different work cycles are consistent with the proposed models, which validate the correctness of the proposed models. Besides, it can be seen from Figure 4 that the system GR shows obvious time-varying effect due to strength degradation. The GR decreases with time. Due to the existence of imperfect maintenance and randomness of the maintenance effect, with the increase of the work cycle, the GR gradually decreases, and the decline speed gradually increases. Therefore, after a certain period of work, the replacement of key components should be considered to avoid the system failure caused by the weakening of the maintenance effect.

It can be known from Figure 5 that the models can directly evaluate the system GFR, which take the system design parameters as the input and can provide the basis for design improvement and fault diagnosis. The GFR of the system reflects the characteristics of conventional bathtub curve. The GFR first decreases and then increases, but there is no constant GFR in the accidental failure period. Thus, the traditional reliability calculation method under the assumption of constant failure rate may introduce large errors. In earlier work cycles, the GFR is low, and the curves are close to a straight line. However, with the increase of work cycles, the characteristics of the bathtub curve are more obvious, and the GFR also increases rapidly with the increase of work cycles, which also shows the necessity of regular replacement strategy for key components. The proposed models can provide guidance for the system maintenance strategy and replacement plan.

To analyze the influences of the failure correlation on the maintenance correlation of mechanical systems, in the case of failure independence (without the HLE), the GR difference (GRD) between the systems with and without the maintenance correlation is shown in Figure 7. Furthermore, the GRD between the systems with and without the maintenance correlation in the case of considering the HLE is also shown in Figure 7.

It can be seen from Figure 7 that the maintenance correlation has considerable impacts on the GR of mechanical systems. The impacts have obvious time-varying characteristics. The maintenance correlation improves the system GR. In each work cycle, the GRD increases with time and then decreases. With the increase of the work cycle, the peak value of the GRD will advance and increase. Meanwhile, it can be seen from Figure 7 that the failure correlation affects the maintenance correlation. When the failure correlation exists in the system, the peak value of the GRD resulting from the maintenance correlation is delayed and reduced. With the increase of the work cycle, the effects of the failure correlation on the GRD become more obvious. In each work cycle, the failure correlation makes the system GRD decrease first and then increase. In general, the failure 
TABLE 1: Discrete distribution function of the MF after the first maintenance.

\begin{tabular}{lcccc}
\hline Possible value & 1 & 0.95 & 0.9 \\
\hline Discrete probability & $1 / 3$ & $1 / 3$ & $1 / 3$ \\
\hline
\end{tabular}

TABLe 2: Discrete distribution function of the MF after the second maintenance.

\begin{tabular}{lccc}
\hline Possible value & 0.95 & 0.9 & 0.85 \\
\hline Discrete probability & $1 / 3$ & $1 / 3$ & $1 / 3$ \\
\hline
\end{tabular}

TABLE 3: Discrete distribution function of MF after the third maintenance.

\begin{tabular}{lccc}
\hline Possible value & 0.9 & 0.85 & 0.8 \\
\hline Discrete probability & $1 / 3$ & $1 / 3$ & $1 / 3$ \\
\hline
\end{tabular}

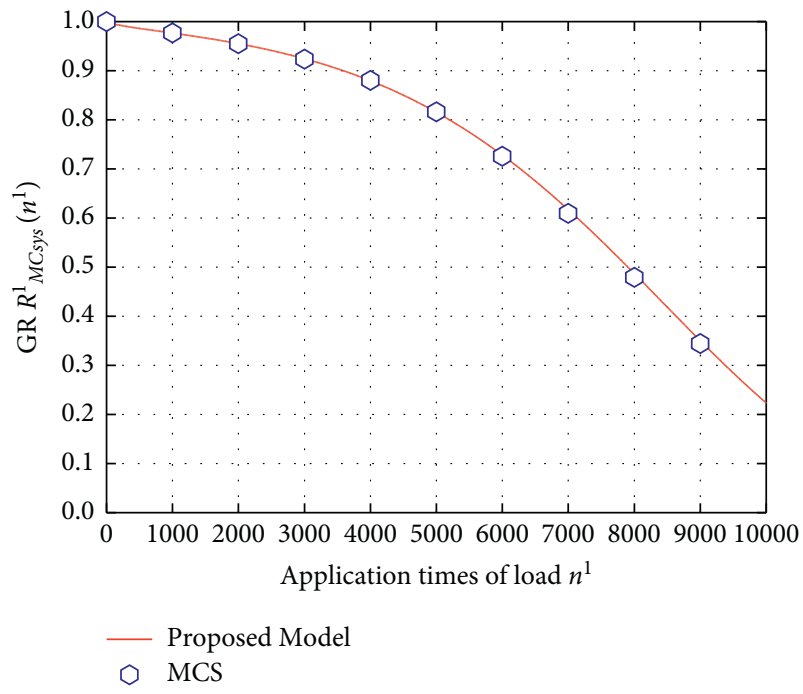

(a)

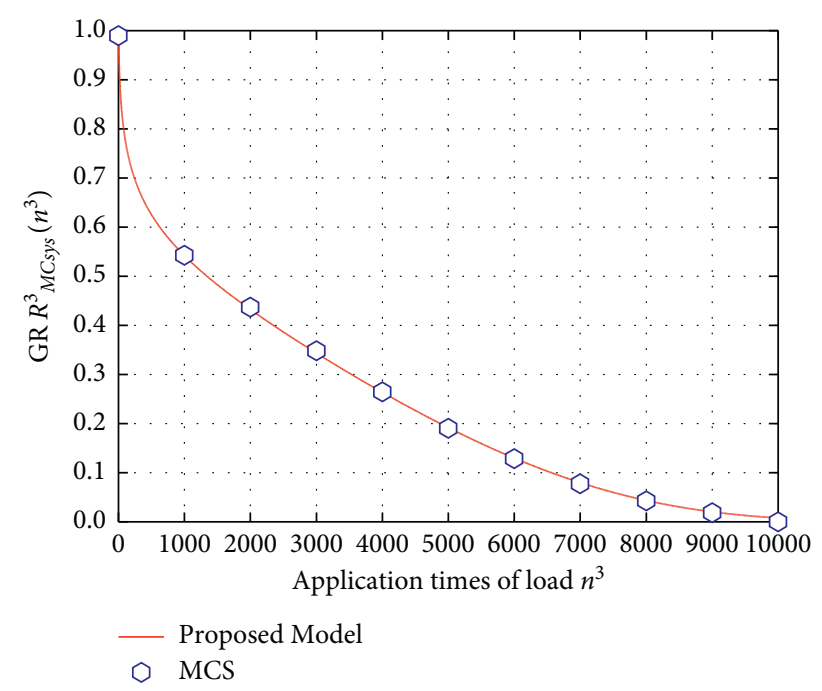

(c)

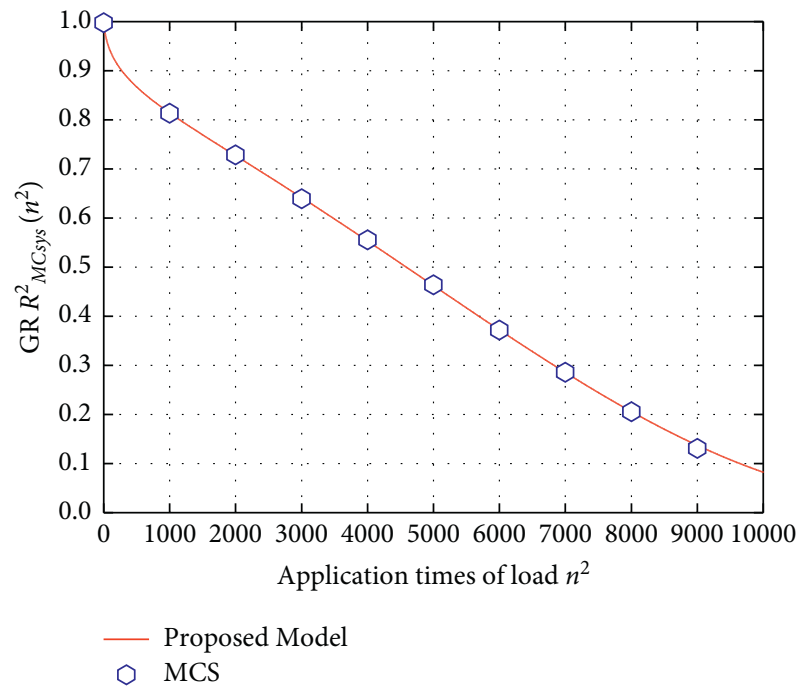

(b)

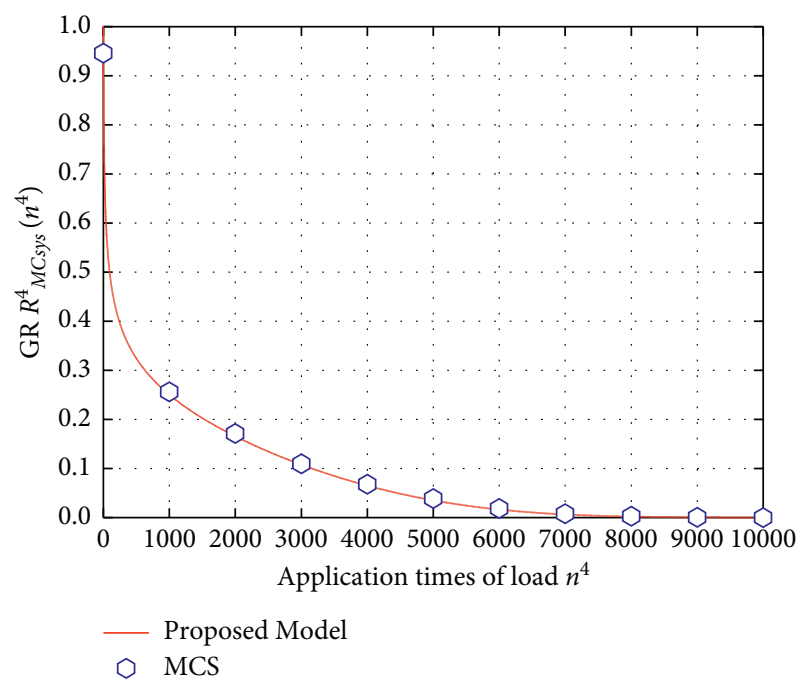

(d)

FIGURE 4: MCS results and the system GR from proposed models in different work cycles. (a) Work cycle 1, (b) work cycle 2, (c) work cycle 3, and (d) work cycle 4 . 


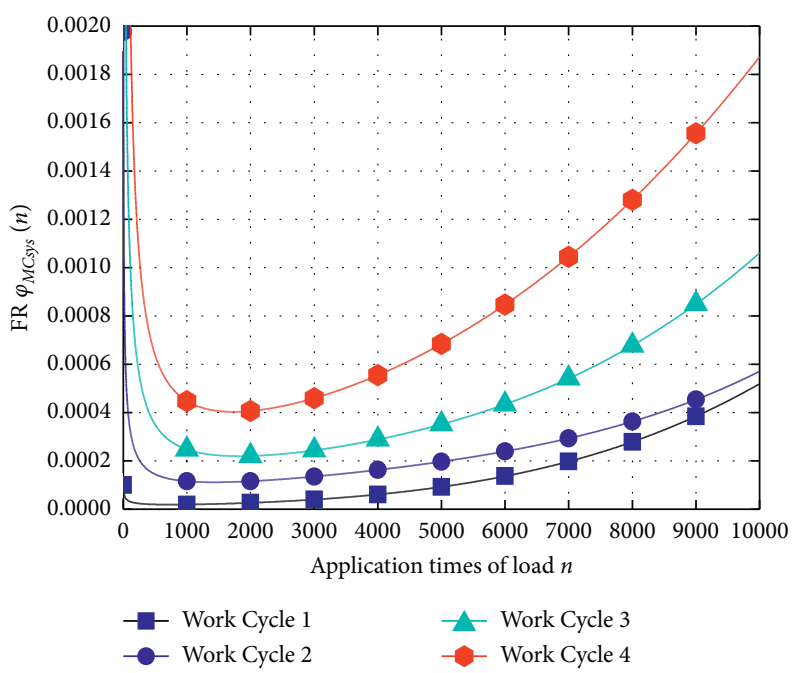

FIGURE 5: System FR in different work cycles.

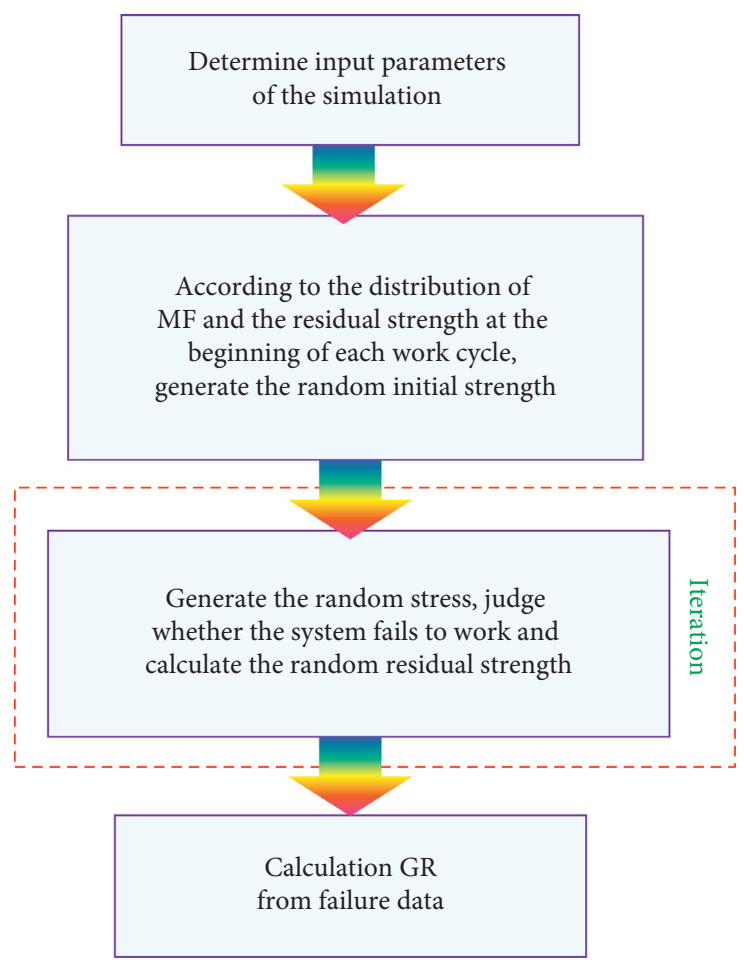

FIgURE 6: MCS flow chart.

correlation suppresses the effects of the maintenance correlation on the system GR.

In order to analyze the impacts of the maintenance correlation on the failure correlation of mechanical systems, in the case of maintenance independence, the GR of the systems with and without the failure correlation is shown in Figure 8 . The GRD between the systems with and without the failure correlation in the case of the maintenance correlation is shown in Figure 8.

It can be known from Figure 8 that the failure correlation has an important influence on the system GR. Similar to the maintenance correlation, the failure correlation improves the system GR, and the extent of improvement increases gradually with time and then decreases gradually. Furthermore, with the increase of the work cycle, the failure correlation makes the peak value of the GRD come earlier. Besides, the maintenance correlation has great impacts on the system failure correlation. The peak value of the GRD caused by the failure correlation is advanced and significantly reduced due to the maintenance correlation. Generally, the maintenance correlation suppresses the influences of the failure correlation on the system GR and makes the 


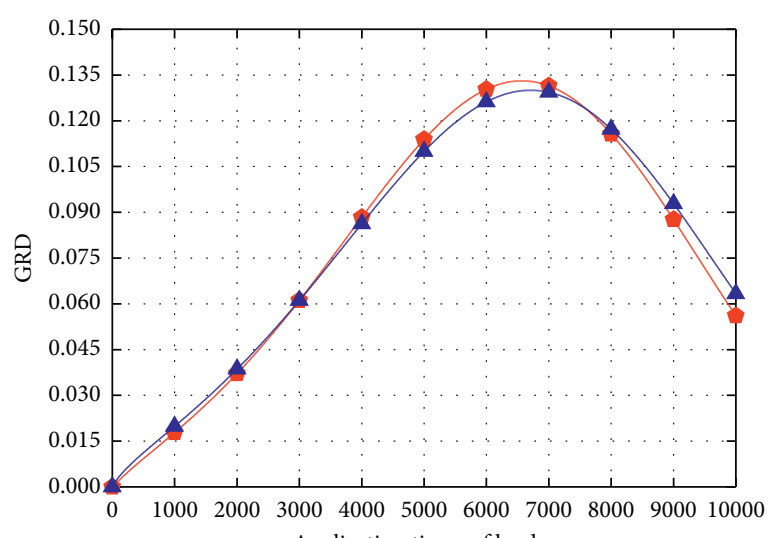
Application times of load $n$

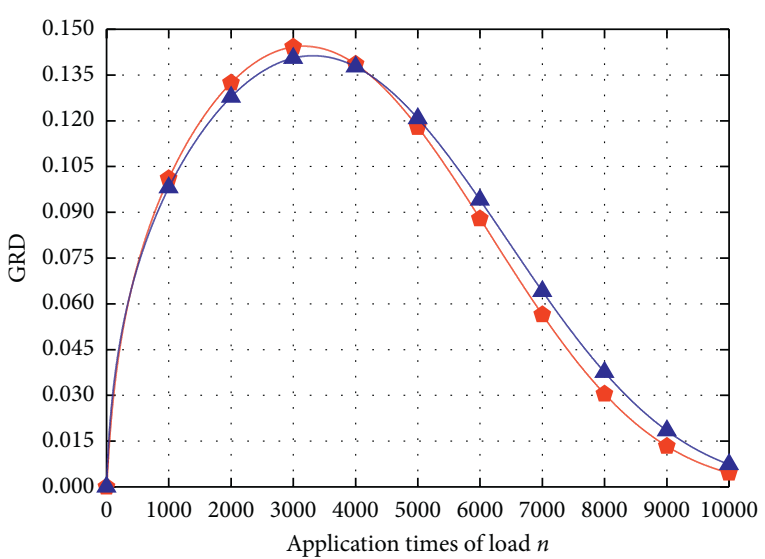

- Independent system

$\rightarrow$ Dependent system

(a)

(b)

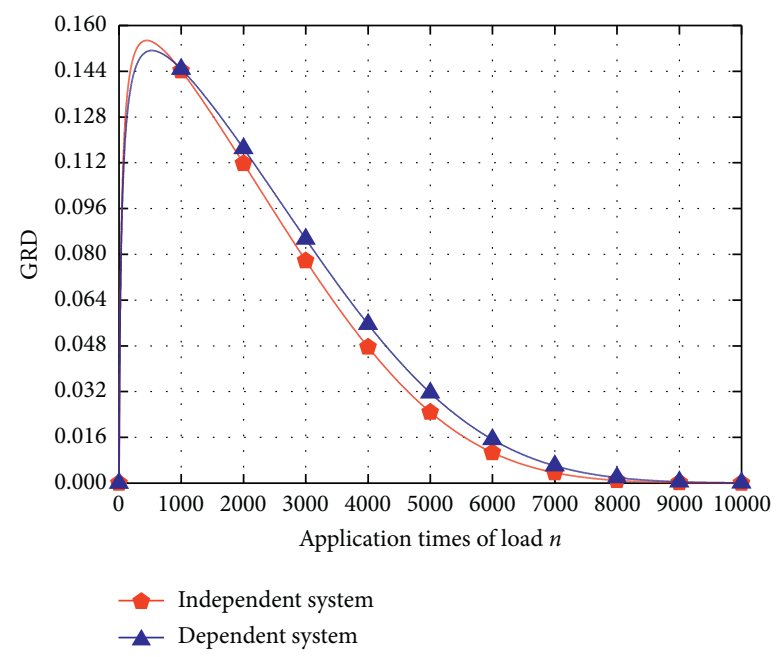

(c)

Figure 7: Comparison of the GRD between the independent system and dependent system. (a) Work cycle 2, (b) work cycle 3, and (c) work cycle 4 .

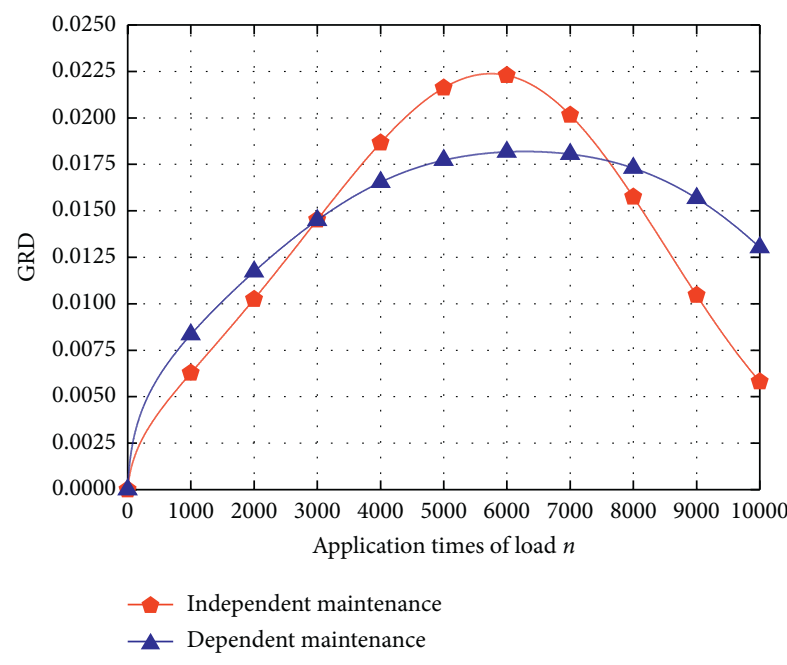

(a)

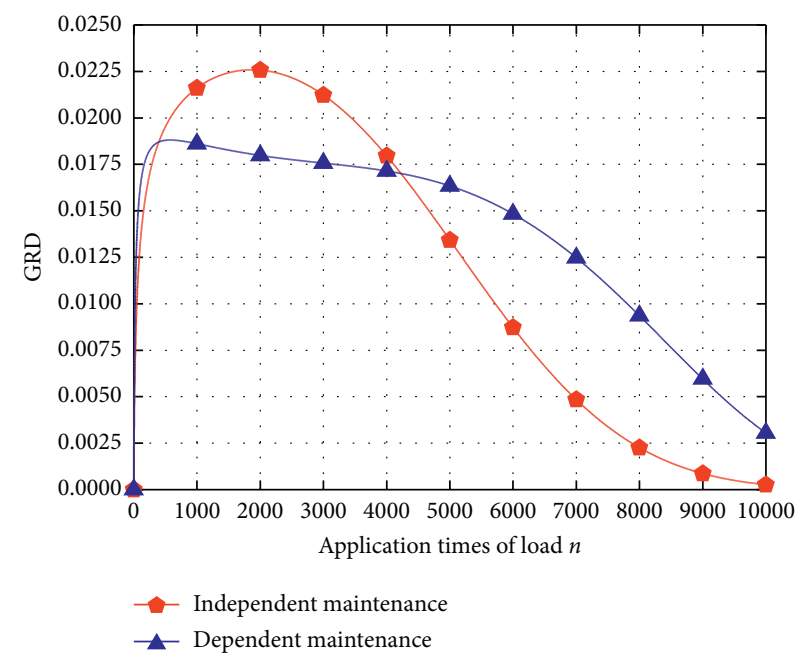

(b)

Figure 8: Continued. 


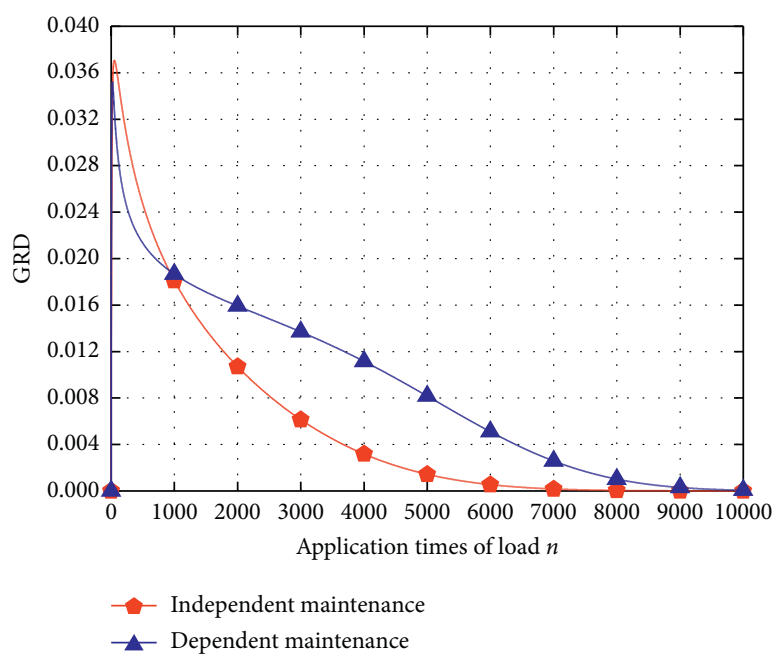

(c)

FIGURE 8: GRD with and without maintenance correlation. (a) Work cycle 2, (b) work cycle 3, and (c) work cycle 4.

influences of the failure correlation on the system GR more stable.

\section{Conclusions}

The proposed reliability models of mechanical systems with imperfect maintenance take the failure correlation caused by the homologous load effect and the maintenance correlation into consideration. The correctness and the effectiveness of the models are verified by MCS. The results from the numerical examples show that the failure correlation on the maintenance correlation have great influences on the system GR. These influences not only have obvious time-varying characteristics in each work cycle but also change with the increase of the work cycle. Besides, the failure correlation and the maintenance correlation also interact with each other, which inhibit each other and change the time-varying characteristics of each other's influences on the system GR in each work cycle and between different work cycles. The proposed models can provide guidance for fault diagnosis and optimization design of mechanical systems.

\section{Data Availability}

The data supporting the findings are included in the study.

\section{Conflicts of Interest}

The authors declare no conflicts of interest.

\section{Acknowledgments}

This work was supported by Scientific Research Funds Project of Liaoning Education Department of China (Grant No. L2019019), FuShun Revitalization Talents Program (Grant No. FSYC202107014), Program for Liaoning Innovative Talents in University (Grant No. LR2017070), and
National Natural Science Foundation of China (Grant No. 51505207).

\section{References}

[1] J. Wu, S. Yan, L. Xie, and P. Gao, "Reliability apportionment approach for spacecraft solar array using fuzzy reasoning Petri net and fuzzy comprehensive evaluation," Acta Astronautica, vol. 76, no. 3, pp. 136-144, 2012.

[2] J. Wu, S. Yan, and M. Zuo, "Evaluating the reliability of multibody mechanisms: a method considering the uncertainties of dynamic performance," Reliability Engineering \& System Safety, vol. 149, no. 5, pp. 96-106, 2016.

[3] J. D. Wang and T. S. Liu, "Fuzzy reliability using a discrete stress-strength interference model," IEEE Transactions on Reliability, vol. 45, no. 1, pp. 145-149, 1996.

[4] Z. An, H. Huang, Z. Wang, X. Zhang, and G. Wang, "Discrete stress-strength interference model of reliability analysis under multi-operating conditions," Chinese Journal of Mechanical Engineering, vol. 23, no. 3, pp. 398-402, 2010.

[5] J. Zhang, X. Ma, and Y. Zhao, "A stress-strength time-varying correlation interference model for structural reliability analysis using copulas," IEEE Transactions on Reliability, vol. 66, no. 2, pp. 351-365, 2017.

[6] R. Barati and S. Setayeshi, "Functional reliability evaluation of an MTR-pool type research reactor core using the load-capacity interference model," Annals of Nuclear Energy, vol. 58, no. 8, pp. 151-160, 2013.

[7] Y. Wu, L. Xie, D. Wang, and J. Gao, "Reliability analysis of shiplift gear based on system-level load-strength interference model," Advanced Materials Research, vol. 118, no. 1, pp. 354-358, 2010.

[8] Z. Yan and G. Li, "Passenger vehicle clutch reliability optimization based on the stress-strength interference model," Applied Mechanics and Materials, vol. 509, no. 1, pp. 86-91, 2014.

[9] H. Guo, B. Suo, and G. Zhang, "Reliability bounds based on universal generating function and discrete stress-strength interference model," International Journal of Plant Engineering and Management, vol. 2017, no. 3, pp. 175-187, 2017. 
[10] Y. Liu, Q. Zhang, Z. Ouyang, and H.-Z. Huang, "Integrated production planning and preventive maintenance scheduling for synchronized parallel machines," Reliability Engineering \& System Safety, vol. 215, no. 2, Article ID 107869, 2021.

[11] Y. Liu, Y. Chen, and T. Jiang, "Dynamic selective maintenance optimization for multi-state systems over a finite horizon: a deep reinforcement learning approach," European Journal of Operational Research, vol. 283, no. 1, pp. 166-181, 2020.

[12] Y. Liu, B. Zhang, T. Jiang, and T. Xiahou, "Optimization of multilevel inspection strategy for nonrepairable multistate systems," IEEE Transactions on Reliability, vol. 69, no. 99, pp. 1-18, 2019.

[13] T. Xia, L. Xi, E. Pan, X. Fang, and N. Gebraeel, "Lease-oriented opportunistic maintenance for multi-unit leased systems under product-service paradigm[J]," Journal of Manufacturing Science and Engineering, vol. 139, no. 7, pp. 1-10, Article ID 071005, 2017.

[14] T. Xia, K. Zhang, B. Sun, X. Fang, and L. Xi, "Integrated remanufacturing and opportunistic maintenance decisionmaking for leased batch production lines[J]," Journal of Manufacturing Science and Engineering, vol. 143, no. 8, pp. 1-35, 2021.

[15] T. Xia, B. Sun, Z. Chen, E. Pan, H. Wang, and L. Xi, “Opportunistic maintenance policy integrating leasing profit and capacity balancing for serial-parallel leased systems[J]," Reliability Engineering \& System Safety, vol. 205, 2021.

[16] Z. Demet, T. Busenur, and S. A. Aksezer, "Cost-effective fault diagnosis of a multi-component dynamic system under corrective maintenance," Applied Soft Computing, vol. 102, no. 3, Article ID 107092, 2021.

[17] M. A. Voicu and I. Fuiorea, "Mean corrective maintenance time for a medium courier turboprop aircraft," Incas Bulletin, vol. 13, no. 1, pp. 237-243, 2021.

[18] A. Ait, E. Cadi, A. Gharbi, K. Dhouib, and A. Artiba, "Joint production and preventive maintenance controls for unreliable and imperfect manufacturing systems," Journal of Manufacturing Systems, vol. 58, no. 1, pp. 263-279, 2021.

[19] V. Rykov, O. Kochueva, and M. Farkhadov, "Preventive maintenance of a k-out-of-n system with applications in subsea pipeline monitoring," Journal of Marine Science and Engineering, vol. 9, no. 1, p. 85, 2021.

[20] H. Jin and M. Rausand, "Reliability of safety-instrumented systems subject to partial testing and common-cause failures," Reliability Engineering \& System Safety, vol. 121, no. 1, pp. 146-151, 2014.

[21] J. K. Vaurio, "Consistent mapping of common cause failure rates and alpha factors," Reliability Engineering \& System Safety, vol. 92, no. 5, pp. 628-645, 2007.

[22] B. R. Ziva and C. Marko, "An extension of Multiple Greek Letter method for common cause failures modelling," Journal of Loss Prevention in the Process Industries, vol. 29, no. 1, pp. 144-154, 2014.

[23] Y. Yang, L. Fang, C. Hou, and L. Yang, "The analysis and evaluation for a multi-unit system maintenance correlation," Journal of Engineering Design, vol. 18, no. 2, pp. 82-86, 2011.

[24] A. Poceski, Fundamentals of the Finite Element Method, Springer, Berlin Heidelberg, 1992.

[25] P. Gao, L. Xie, and F. Liu, "Dynamic reliability analysis of series mechanical systems considering strength degradation path dependence of components," Journal of Advanced Mechanical Design, Systems, and Manufacturing, vol. 9, no. 5, pp. 1-13, 2015. 